\title{
Análisis Bibliométrico de las Revistas Iberoamericanas más Relevantes Afines a la Psicología Clínica y Salud del Journal Citation Reports (2011)
}

\section{Bibliometric Analysis of the most relevant Iberoamerican Journals related to Clinical and Health Psychology of the Journal Citation Reports (2011)}

\author{
Tania Ariza \\ M. Reina Granados \\ Universidad de Granada, España
}

(Rec: 30 de Julio de 2012 / Acep: 01 de octubre de 2012)

\begin{abstract}
Resumen
Las revistas científicas de psicología son el punto de referencia de la investigación teórica y metodológica. Por ello, el objetivo del presente estudio es realizar un análisis bibliométrico de International Journal of Clinical and Health Psychology, Revista Argentina de Clínica Psicológica, Revista Latinoamericana de Psicopatología Fundamental, Salud Mental y Terapia Psicológica de los últimos cinco años. El análisis de los artículos se hizo a partir de indicadores del Journal Citation Reports (factor de impacto, el índice de inmediatez y número de citas), e indicadores bibliométricos (número total de artículos, índice de autoría, filiación institucional de los autores, metodología, tipo de muestra, temática general y específica e idioma de publicación). En los resultados se comprueban las características de cada revista y finalmente, se reflexiona sobre los aspectos positivos que las revistas deberían mantener y los aspectos negativos que se pueden mejorar para que logren un mayor prestigio.

Palabras clave: bibliometría, revistas, psicología, factor de impacto, Journal Citation Reports.
\end{abstract}

\begin{abstract}
Psychology journals are the benchmark of theoretical and methodological research. Therefore, the purpose of the current study consisted of conducting a bibliometric analysis of International Journal of Clinical and Health Psychology, Revista Argentina de Clínica Psicológica, Revista Latinoamericana de Psicopatología Fundamental, Salud Mental and Terapia Psicológica for the past five years. Content analysis of articles was based on indicators of the Journal Citation Reports (impact factor, immediacy index and number of citations) and bibliometric indicators (number of articles, authorship index, institutional affiliation, methodology, types of sample, general and specific themes and language of publication). Our results verify the characteristics of each journal and finally, it reflects on the positive aspects that should be maintained by the journals and the negative aspects that can be improved to achieve greater prestige.

Keywords: bibliometrics, journals, psychology, impact factor, Journal Citation Reports.
\end{abstract}

\section{Introducción}

Las revistas científicas de psicología son el punto de referencia documental en temas concretos de investigación teórica y metodológica en ese ámbito. En las últimas décadas, esta disciplina ha crecido de manera exponencial (Cortada de Kohan, 2002), lo que ha originado, a su vez, el avance en las Ciencias Sociales de la Bibliometría (Buela-Casal, Olivas-Ávila, Musi-Lechuga y Zych, 2011; Pritchard, 1969; Quevedo-Blasco, Ariza y Raya, 2012) que se utiliza para medir la actividad, estructura, evolución y repercusión de estas publicaciones (Quevedo-Blasco y López-López, 2010). A través de un método objetivo y eficaz como es el uso de indicadores bibliométricos (Zych y QuevedoBlasco, 2011) se obtienen resultados válidos que aportan un conocimiento nuevo, permitiendo así la identificación de aspectos relevantes de la situación científica, como las áreas temáticas prioritarias, las disciplinas emergentes y el volumen de producción territorial (Agudelo, Buela-Casal y Bretón-López, 2003, 2004; Buela-Casal, Bermúdez, Sierra, Quevedo-Blasco y Castro, 2010; Buela-Casal et al., 2011, 2012; Buela-Casal, Bermúdez, Sierra, Quevedo-Blasco,

Correspondencia: Tania Ariza. Facultad de Psicología, Universidad de Granada. Campus de Cartuja, s/n, 18011. Granada, España. E-mail: tariza@ugr.es 
Guillén-Riquelme et al., 2010, 2012; Zubeidat, Desvarieux, Salamanca y Sierra, 2004), entre otras.

Las bases de datos, especializadas y multidisciplinares, son las fuentes de información más utilizadas para la difusión de la labor científica, ya que en éstas aparecen publicaciones de las fuentes primarias de información, se almacenan de forma sistemática datos bibliográficos y permiten conseguir indicadores bibliométricos (Alvis-Guzmán y De La HozRestrepo, 2002). Entre los indicadores mencionados cabe destacar los índices de citas que reciben los trabajos publicados en revistas incluidas en el Journal Citation Reports (JCR), que indican el número de veces que un artículo o un autor es citado, así como el Factor de Impacto (FI) de una revista, que representa el número total de citas que reciben en un año los artículos publicados en la misma en los dos años anteriores entre el número de artículos publicados en esos dos años (Buela-Casal, 2003; Garfield, 2003; GómezGarcía, Ramiro, Ariza y Granados, 2012; Quevedo-Blasco, Díaz-Piedra y Guglielmi, 2010), entre otros (Villar, 2011).

A partir de la base de datos JCR se han realizado artículos en los que se comparan publicaciones periódicas y se evalúa la importancia de las revistas dentro de las disciplinas que conforman la psicología (Agudelo et al., 2003; Zych y Buela-Casal, 2007). El FI se suele tener en cuenta en la mayoría de los trabajos bibliométricos (Agudelo et al., 2004; Villalobos-Galvis y Puertas-Campanario, 2007; Zych y Buela-Casal, 2010), concibiéndose la idea de que un alto impacto significa una mayor calidad, opinión que está siendo cuestionada en los últimos años, ya que este factor no avala de forma absoluta la excelencia de los artículos publicados en una revista (Buela-Casal, 2003).

Las revistas científicas son las vías de difusión más utilizadas entre investigadores, por lo que la evaluación de diferentes parámetros bibliométricos (Buela-Casal, 2010), como los tipos de estudio, las temáticas más utilizadas o el idioma de divulgación de los trabajos publicados, entre otros indicadores (e.g., Zych y Quevedo-Blasco, 2011), es decisivo para el avance de una ciencia de calidad. En España y Latinoamérica, se han incorporado trabajos basados en la Cienciometría en la investigación del campo de la psicología (e.g., Navarrete-Cortés, Fernández-López, López-Baena, Quevedo-Blasco y Buela-Casal, 2010; Navarrete-Cortés, Quevedo-Blasco, Chaichio-Moreno, Ríos y Buela-Casal, 2009; Olivas-Ávila y Musi-Lechuga, 2012), debido a la importancia que tiene el conocimiento de la estructura y dinámica de estas revistas (Gallegos, 2010; García-Sílberman, Arana, Martínez, Infante y Jiménez, 2004; Granados, Ariza, Gómez-García y Ramiro, 2011). De hecho, la evaluación de las revistas iberoamericanas de psicología es posible mediante la utilización de índices bibliométricos (e.g., Quevedo-Blasco y López-López, 2011).

Por ejemplo, Agudelo et al. $(2003,2004)$ realizaron análisis bibliométricos comparando revistas en castellano de psicología clínica y psicología de salud, como la Revista
Argentina de Clínica Psicológica (RACP), Salud Mental o International Journal of Clinical and Health Psychology (IJCHP). En esta línea, Villalobos-Galvis y Puertas-Campanario (2007) estudiaron el impacto y la internacionalidad de tres revistas iberoamericanas de psicología, entre las que se encuentra esta última revista. Además, fue medida su internacionalidad en un estudio de Buela-Casal, Perakakis, Taylor y Checa (2006) y una década de la revista en Zych y Quevedo-Blasco (2011).

En otros trabajos, como el de Vera-Villarroel y Mustaca (2006), se analizan las investigaciones de psicología clínica basada en la evidencia de Chile y Argentina de las revistas Terapia Psicológica y la RACP. Ortiz y Vera-Villaroel (2003) realizaron un análisis bibliométrico con revistas chilenas de psicología, donde se analiza, entre otras, una década de Terapia Psicológica. La Revista Latinoamericana de Psicopatología Fundamental (RLPF) también es analizada en el estudio de Quevedo-Blasco y López-López (2010).

Dada la importancia del análisis de la producción científica a través de indicadores bibliométricos, el presente trabajo tiene como objetivo realizar un análisis de cinco revistas afines a la Psicología Clínica y de la Salud. Para este fin, se consideran indicadores utilizados con anterioridad por Agudelo et al. (2004), Zych y Buela-Casal (2010), y Zych y Quevedo-Blasco (2011) en estudios bibliométricos. Para la realización de este estudio se han tenido en cuenta las recomendaciones de Hartley (2012) en cuanto a las técnicas de estructura y redacción. Con esta investigación se pretende conocer las características y evolución en los últimos cinco años de las revistas más relevantes del JCR, con un enfoque en psicología clínica y salud.

\section{Método}

\section{Unidad de análisis}

Se analizaron los artículos publicados entre 2007 y 2011 de las cinco revistas iberoamericanas más relevantes del ámbito de la Psicología Clínica y de la Salud e indexadas en el JCR de 2011. Estas revistas contienen en el título alguna de las palabras: "clínica", "salud", "psicopatología" o "terapia" (en español o inglés). En aquellos casos en los que más de una revista perteneciente al mismo país cumplía estos criterios, se seleccionó la de mayor FI en el JCR. En concreto, las revistas fueron:

- International Journal of Clinical and Health Psychology (España).

- Revista Argentina de Clínica Psicológica (Argentina).

- Revista Latinoamericana de Psicopatología Fundamental (Brasil).

- Salud Mental (México).

- Terapia Psicológica (Chile).

Los artículos de cada revista se analizaron considerando los siguientes indicadores (véase Tabla 1): 


\section{Materiales}

La revista International Journal of Clinical Health Psychology (ISSN 2174-0852 versión online e ISSN 16972600 versión impresa) es editada por la Asociación Española de Psicología Conductual (AEPC) desde el año 2001. Se trata de una publicación cuatrimestral, cuyos trabajos tanto teóricos como experimentales son publicados en inglés y excepcionalmente en español. Esta revista está incluida en la Web of Science (WoS) desde 2005, en concreto, en el Social Sciences Citation Index e indexada en Social Scisearch, Current Contents/Social y Behavioral Sciences, JCR/Social Sciences Edition, PsycINFO, Scopus, Elsevier Bibliographic Databases, EBSCO Publishing, ProQuest Pychology Journals, National Library of Medicine, DOAJ Directory of Open Access Journals, Ulrich International Periodical Directory, Open J-Gate, IN-RECS, ISOC, PSICODOC, Compludoc, Psyke, RedAlyc, Latindex, Biblioteca Nacional de Ciencias de la Salud y Dialnet. Además, cuenta con un consejo editorial de expertos reconocidos mundialmente. Los contenidos están dirigidos al ámbito de la psicología clínica y la salud.
La Revista Argentina de Clínica Psicológica (ISSN 0327-6716 versión impresa e ISSN 1851-7951 versión electrónica) es editada desde 1992 por la Fundación Aiglé (Argentina). Anualmente son publicados tres números en versión impresa y electrónica dirigidos principalmente a profesionales del área de Salud Mental. Los idiomas de difusión son el español y el inglés. Esta revista está en la WoS desde 2007, concretamente en el Science Citation Index Expanded y el Social Sciences Citation Index, y está incluida en otras bases de datos científicas: Social Scisearch, JCR/ Social Sciences Edition, JCR/ Sciences Edition, PsyInfo, Latindex, LILACS, PSICODOC, E-Psyche database, Clase, Dialnet, EBSCO, SCOPUS y RedAlyc.

La Revista Latinoamericana de Psicopatología Fundamental (ISSN 1415-4714 versión impresa e ISSN 1984-0381 versión electrónica), desde el año 1998 es editada en Brasil de forma trimestral por la Associação Universitária de Pesquisa em Psicopatologia Fundamental. En esta revista son publicados trabajos en cuatro idiomas (portugués, español, francés e inglés) y está incluida en la WoS desde 2008, en concreto en el Social Sciences Citation Index y en distintas bases de datos bibliográficas como

Tabla 1. Descripción de los indicadores analizados.

\begin{tabular}{ll}
\hline Indicadores & Descripción \\
\hline JCR & \\
\hline
\end{tabular}

Evolución del Factor de Impacto (FI)

Índice de inmediatez de 2011

Número de citas

Bibliométricos

Número de documentos

Índice de autoría

Documentos por países

Metodología

Muestra

Temáticas

Idioma depublicación
Distribución de las revistas en función del Factor de Impacto que ha tenido cada una en los diferentes años desde su indexación en el Journal Citation Reports (JCR) hasta la actualidad (2011).

Número de citas de 2011 en los artículos publicados en la revista en este año, entre el número de documentos publicados en 2011.

Número de citas que tienen las revistas analizadas en la Web of Science (WoS) en el JCR. 
Social Scisearch y JCR/Social Sciences Edition BVS-Psi, LILACS/BIREME, Clase, PSICODOC, QUALIS. En ella participan profesores y científicos de universidades públicas y privadas de todo el mundo.

La revista Salud Mental (ISSN 0185-3325 versión impresa) fue fundada en 1977 y pertenece al Instituto Nacional de Psiquiatría Ramón de la Fuente Muñiz (México). Se puede encontrar tanto en versión impresa como electrónica y sus artículos están disponibles en lengua castellana o inglesa. Normalmente, se publican seis números al año, excepto determinadas ocasiones en las que se han publicado suplementos o algún número especial. Está indexada en la WoS desde 1985, concretamente está en Social Sciences Citation Index y además está incluida en otras bases como Current Contents / Social and Behavioral Sciences, JCR, LILACS-BIREME, Psychological Abstracts, PsycINFO, IMBIOMED, ASCATOPICS, SSALUD-CENIDS, Bowker Serials Bibliographies, ARTEMISA-SSA, EMBASE Psychiatry, EMBASE Neurosciences, LanPsiquiatría, EBSCO host, PSICODOC, IRESIE-UNAM, CAMEX-UNAM, CONACYT, RedAlyc y Salud Mental en Línea.

La revista Terapia Psicológica se fundó en 1982 por la Sociedad Chilena de Psicología Clínica. Está disponible en versión impresa (ISSN 0716-6184) y electrónica (ISSN 0718-4808), y se publican tres números al año, en los meses de abril, julio y diciembre, tanto en castellano como en inglés. Además, está indexada en diversas bases de datos reconocidas a nivel internacional como en la WoS desde 2009, JCR, DIALNET, DOAJ, EMBASE/Excerpta Médica, INDEX COPERNICUS, Latindex, Biblioteca Virtual en Salud, PSICODOC, APA Online, RedAlyc, SciELO Chile, Scopus, PsicINFO y LILACS-BIREME. Esta revista publica artículos originales, revisiones e informes breves relacionados con la Psicología Clínica, dirigidos a profesionales e investigadores de este campo de conocimiento. El Comité Editorial está formado por expertos de distintos países iberoamericanos así como de Estados Unidos.

\section{Diseño y procedimiento}

Se trata de un estudio descriptivo mediante análisis de documentos. En primer lugar, se realizó una selección de los trabajos publicados en las revistas anteriormente mencionadas y solamente se consideraron los artículos originales y las revisiones. La obtención de información se hizo utilizando la versión electrónica de las revistas y para lograr una mayor fiabilidad de los datos, el análisis se realizó por dos investigadores de forma independiente durante los meses de mayo y junio de 2012. Posteriormente, se hizo la puesta en común y el grado de acuerdo entre ellos fue superior al $97 \%$.

Para el análisis de las revistas se consideraron indicadores relacionados con el JCR (Factor de Impacto de 2011, índice de inmediatez y número de citas) e indicadores bibliométricos (número de documentos, índice de autoría, productividad por país, tipo de metodología utilizada, tipo de muestra empleada, temática general y específica e idioma de publicación).

El indicador relacionado con el número de documentos hace referencia al número de artículos publicados en cada una de las revistas desde 2007 a 2011. El índice de autoría representa el porcentaje de contribuciones según el número de autores que firma el artículo. Respecto a la productividad por país, se consideró la filiación institucional de todos los autores que aparecían en cada artículo. En cuanto a la metodología utilizada en los estudios se siguió la siguiente clasificación: teóricos (clásicos y meta-análisis), empíricos (descriptivos, ex post facto, experimentales, cuasi-experimentales, instrumentales y experimentos de caso único), y estudios cualitativos (estudios de caso, etnografías y estudios de investigación-acción).

El tipo de muestra se clasificó en función de los siguientes grupos: a) revistas, b) sin muestra/teórico, c) familia/padres, d) profesionales, e) niños, f) adolescentes, g) adultos y h) ancianos. El grupo de niños, adolescentes y adultos se dividió en dos subgrupos (con y sin patología). Se añadió un grupo llamado "otro" cuando el tipo de muestra no correspondía con ninguno de los anteriores. Para la clasificación de los artículos en función de la temática se siguió la nomenclatura internacional de la UNESCO (1988) en el campo de la psicología, considerando las diferentes disciplinas para la temática general (véanse criterios de inclusión en la Tabla 2) y sus correspondientes subdisciplinas para la temática específica. Por último, también se realizó un análisis de los artículos según el idioma de publicación.

\section{Resultados}

En este estudio bibliométrico se analizan las características de la producción científica de IJCHP, RACP, $R L P F$, Salud Mental y Terapia Psicológica, para conocer sus características y evolución en los últimos cinco años.

En la Tabla 3 puede apreciarse la evolución del FI de las revistas analizadas y otros datos pertenecientes al JCR de 2011. Se puede comprobar que IJCHP es la revista que cuenta con un mayor FI, seguida de Terapia Psicológica. En cambio, la que tiene un mayor índice de inmediatez es la $R A C P$. En cuanto al número de citas recibidas por las revistas, IJCHP está en primera posición, siendo Salud Mental la que se encuentra en segundo lugar.

En la Tabla 4 se puede observar el número de artículos que fueron analizados de cada revista, así como el idioma de publicación de los mismos. La RACP publicó la totalidad de sus artículos en español. También, Salud Mental y Terapia Psicológica publicaron la mayor parte de sus artículos en español, mientras que en IJCHP más de la mitad de sus artículos están escritos en lengua inglesa. Por otra parte, el $84.3 \%$ de los artículos de la $R L P F$ están en portugués. 
Tabla 2. Criterios de inclusión de los artículos en las temáticas generales de la UNESCO (1988).

\begin{tabular}{|c|c|}
\hline Temática general & Criterios \\
\hline Patología & $\begin{array}{l}\text { Se incluyen artículos sobre desórdenes del comportamiento, discapacidad inte- } \\
\text { lectual y psicopatología. }\end{array}$ \\
\hline Psicología del niño y del adolescente & $\begin{array}{l}\text { Se incluyen artículos sobre psicología evolutiva y escolar, problemas de apren- } \\
\text { dizaje y patología del lenguaje. }\end{array}$ \\
\hline Asesoramiento y orientación & $\begin{array}{l}\text { Se incluyeron artículos sobre terapia del comportamiento y de grupo, psicología } \\
\text { de la orientación, psicoanálisis y psicoterapia. }\end{array}$ \\
\hline Psicopedagogía & Se incluyeron artículos sobre los procesos cognitivos y los métodos educativos. \\
\hline Evaluación y diagnóstico en psicología & $\begin{array}{l}\text { Se incluyeron artículos sobre psicología diferencial, teoría de la medición, esta- } \\
\text { dística, psicometría, análisis de la escala, elaboración y validez de test. }\end{array}$ \\
\hline Psicología experimental & $\begin{array}{l}\text { Se incluyeron artículos sobre actividad cerebral, psicología comparada y fisio- } \\
\text { lógica, emoción, reacción y reflejos, experimentos sobre la conducta, niveles de } \\
\text { actividad, procesos sensoriales, mentales y de la memoria. }\end{array}$ \\
\hline Psicología general & Se incluyeron artículos sobre las corrientes teóricas en psicología. \\
\hline Psicología de la vejez & Se incluyeron artículos sobre la madurez, la senectud y la muerte. \\
\hline Psicología industrial & $\begin{array}{l}\text { Se incluyeron artículos sobre prevención de accidentes, motivación y comporta- } \\
\text { miento en el trabajo, planificación de puestos de trabajo, selección de personal } \\
\text { y evaluación del rendimiento. }\end{array}$ \\
\hline Parapsicología & Se incluyeron artículos sobre la percepción extrasensorial y la hipnosis. \\
\hline Personalidad & $\begin{array}{l}\text { Se incluyeron artículos sobre la creatividad, la cultura y el desarrollo, medida, } \\
\text { estructura y teoría de la personalidad. }\end{array}$ \\
\hline Estudio psicológico social & Se incluyeron artículos sobre discriminación y fenómenos de grupos minoritarios. \\
\hline Psicofarmacología & $\begin{array}{l}\text { Se incluyeron artículos sobre alcoholismo, reacciones del comportamiento y el } \\
\text { uso/abuso de drogas así como su tratamiento. }\end{array}$ \\
\hline Psicología social & $\begin{array}{l}\text { Se incluyeron artículos sobre actitudes, comportamiento colectivo, psicología } \\
\text { comunitaria, resolución de conflictos, psicología forense, marketing, comporta- } \\
\text { miento colectivo y opinión pública. }\end{array}$ \\
\hline Otras especialidades psicológicas & Se incluyeron artículos cuya temática no pertenecía a ninguna de las anteriores. \\
\hline
\end{tabular}

En cuanto al índice de autoría, cabe decir que los artículos de un autor destacan en la $R L P F(61.5 \%)$ y la $R A C P(31.7 \%)$. En Terapia Psicológica, la mayor parte de los artículos fueron firmados por dos autores (35.4\%). En IJCHP y Salud Mental prevalecen los artículos escritos por tres $(29.1 \%)$ y cuatro autores (26.1\%), respectivamente (véanse Tablas 5, 6, 7, 8 y 9).

Al comprobar la filiación institucional de los autores que publican artículos en las revistas analizadas, los resultados pusieron de manifiesto que en todas publican autores que pertenecen a instituciones de distintos países iberoamericanos (véase Tabla 10).

La metodología de los artículos de las revistas es muy variada (véase Tabla 11). Los artículos teóricos predominan en la $R L P F(87.7 \%)$ y la $R A C P(51.3 \%)$. Entre este tipo de artículos prevalecen los estudios clásicos y representan una mínima parte los meta-análisis. Los artículos con una metodología empírica destacan en IJCHP (83.4\%), Terapia Psicológica (63.6\%) y Salud Mental (54.5\%). Entre los subtipos de estudios empíricos, en IJCHP predominan los ex post facto, en Terapia Psicológica los instrumentales y en Salud Mental aquellos que siguen una metodología descriptiva. Los artículos cualitativos representan la menor parte en todas las revistas analizadas.

El $35.4 \%$ de los artículos publicados en Terapia Psicológica tienen una muestra formada por adultos, entre los que predominan aquellos que no tienen ninguna patología. En IJCHP (56.5\%), la RACP (44.4\%) y Salud Mental (29.5\%) también prevalecen los artículos sobre adultos, en 
Tabla 3. Características de las revistas en relación a la evolución de su FI (2007-2011), Índice de Inmediatez (2011) y Número de Citas en el Journal Citation Reports.

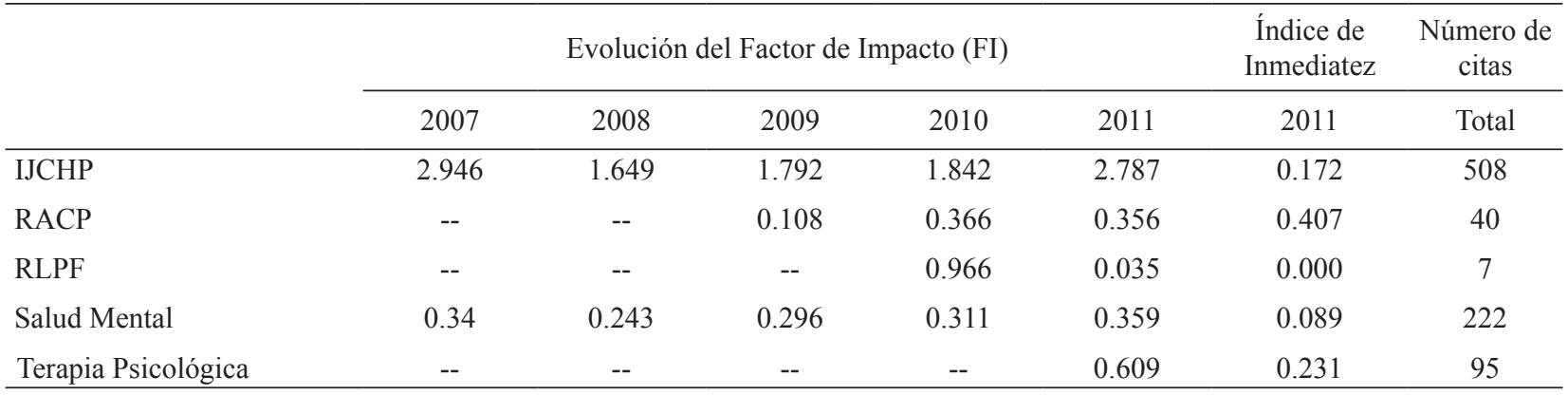

Nota. IJCHP = International Journal of Clinical and Health Psychology; RACP = Revista Argentina de Clínica Psicológica; RLPF = Revista Latinoamericana de Psicopatología Fundamental.

Tabla 4. Número de artículos y porcentaje según el idioma de publicación en cada una de las revistas entre 2007 y 2011.

\begin{tabular}{lccccc}
\hline & Número de artículos & \multicolumn{3}{c}{ Idioma de publicación } \\
\cline { 2 - 6 } & & Español & Inglés & Francés & Portugués \\
\hline IJCHP & 175 & 38.9 & 60.6 & 0 & 0.6 \\
RACP & 115 & 100 & 0 & 0 & 0 \\
RLPF & 178 & 6.7 & 3.9 & 5.1 & 84.3 \\
Salud Mental & 257 & 86.3 & 13.6 & 0 & 0 \\
Terapia Psicológica & 99 & 94.9 & 5.1 & 0 & 0 \\
\hline
\end{tabular}

Nota. IJCHP = International Journal of Clinical and Health Psychology; RACP = Revista Argentina de Clínica Psicológica; RLPF = Revista Latinoamericana de Psicopatología Fundamental.

Tabla 5. Porcentaje de artículos publicados en International Journal of Clinical and Health Psychology, en función del Índice de Autoría (2007-2011).

\begin{tabular}{|c|c|c|c|c|c|c|c|}
\hline \multicolumn{8}{|c|}{ Número de autores } \\
\hline & 1 & 2 & 3 & 4 & 5 & 6 & 7 o más \\
\hline Años & & & & $\%$ & & & \\
\hline 2007 & 2.2 & 22.2 & 37.8 & 15.6 & 13.3 & 4.4 & 4.4 \\
\hline 2008 & 2.2 & 17.8 & 35.6 & 22.2 & 15.6 & 4.4 & 2.2 \\
\hline 2009 & 0 & 16.7 & 40.0 & 20.0 & 13.3 & 6.7 & 3.3 \\
\hline 2010 & 7.1 & 3.6 & 7.1 & 39.3 & 10.7 & 21.4 & 10.7 \\
\hline 2011 & 11.1 & 37.0 & 14.8 & 14.8 & 11.1 & 3.7 & 7.4 \\
\hline$\%$ Total & 4.0 & 19.4 & 29.1 & 21.7 & 13.1 & 7.4 & 5.1 \\
\hline
\end{tabular}

Tabla 6. Porcentaje de artículos publicados en la Revista Argentina de Clínica Psicológica, en función del Índice de Autoría (2007-2011).

\begin{tabular}{lccccccc}
\hline \multicolumn{1}{c}{ Número de autores } \\
\hline Años & 1 & 2 & 3 & 4 & 5 & 6 & 7 o más \\
\hline & & & $\%$ & & & \\
2007 & 54.2 & 25.0 & 16.7 & 0 & 4.2 & 0 & 0 \\
2008 & 61.1 & 16.7 & 0 & 5.6 & 16.7 & 0 & 0 \\
2009 & 0 & 13.6 & 40.9 & 9.1 & 18.2 & 9.1 & 9.1 \\
2010 & 25.0 & 20.8 & 29.2 & 16.7 & 8.3 & 0 & 5 \\
2011 & 18.5 & 33.3 & 29.6 & 3.7 & 0 & 7.4 & 7.4 \\
\% Total & 30.4 & 22.6 & 24.3 & 7.0 & 8.7 & 3.5 & 3.5 \\
\hline
\end{tabular}


Tabla 7. Porcentaje de artículos publicados en la Revista Latinoamericana de Psicopatología Fundamental, en función del Índice de Autoría (2007-2011).

\begin{tabular}{|c|c|c|c|c|c|c|c|c|}
\hline \multicolumn{9}{|c|}{ Número de autores } \\
\hline \multirow[b]{2}{*}{ Años } & 1 & 2 & 3 & & & & 6 & 7 o más \\
\hline & \multicolumn{8}{|c|}{$\%$} \\
\hline 2007 & 77.4 & 19.3 & 0 & 3.2 & 0 & 0 & & 0 \\
\hline 2008 & 77.2 & 15.9 & 2.2 & 4.5 & 0 & 0 & & 0 \\
\hline 2009 & 65.8 & 26.8 & 0 & 4.8 & 2.4 & 0 & & 0 \\
\hline 2010 & 42.4 & 24.2 & 21.2 & 6.0 & 0 & 0 & & 6.0 \\
\hline 2011 & 44.8 & 37.9 & 10.3 & 3.4 & 3.4 & 0 & & 0 \\
\hline$\%$ Total & 62.9 & 24.2 & 6.2 & 4.5 & 1.1 & 0 & & 1.1 \\
\hline
\end{tabular}

Tabla 8. Porcentaje de artículos publicados en Salud Mental, en función del Índice de Autoría (2007-2011).

\begin{tabular}{|c|c|c|c|c|c|c|c|}
\hline \multicolumn{8}{|c|}{ Número de autores } \\
\hline \multirow[b]{2}{*}{ Años } & 1 & 2 & 3 & 4 & 5 & 6 & 7 o más \\
\hline & \multicolumn{7}{|c|}{$\%$} \\
\hline 2007 & 11.3 & 15.1 & 11.3 & 32.1 & 18.9 & 5.7 & 5.7 \\
\hline 2008 & 4.3 & 17.0 & 31.9 & 27.7 & 4.3 & 6.4 & 8.5 \\
\hline 2009 & 5.8 & 13.5 & 19.2 & 23.1 & 13.5 & 5.8 & 19.2 \\
\hline 2010 & 13.2 & 17.0 & 15.1 & 26.4 & 11.3 & 7.5 & 9.4 \\
\hline 2011 & 3.8 & 15.4 & 17.3 & 21.2 & 19.2 & 11.5 & 11.5 \\
\hline$\%$ Total & 7.8 & 15.6 & 18.7 & 26.1 & 13.6 & 7.4 & 10.9 \\
\hline
\end{tabular}

Tabla 9. Porcentaje de artículos publicados en Terapia Psicológica, en función del Índice de Autoría (2007-2011).

\begin{tabular}{|c|c|c|c|c|c|c|c|}
\hline \multicolumn{8}{|c|}{ Número de autores } \\
\hline \multirow[b]{2}{*}{ Años } & 1 & 2 & 3 & 4 & 5 & 6 & 7 o más \\
\hline & \multicolumn{7}{|c|}{$\%$} \\
\hline 2007 & 15.8 & 31.6 & 21.1 & 10.5 & 10.5 & 10.5 & 0 \\
\hline 2008 & 20 & 40 & 10 & 15 & 5 & 10 & 0 \\
\hline 2009 & 10 & 25 & 20 & 20 & 15 & 5 & 5 \\
\hline 2010 & 15 & 45 & 5 & 20 & 10 & 0 & 5 \\
\hline 2011 & 20 & 30 & 15 & 25 & 5 & 0 & 5 \\
\hline$\%$ Total & 16.2 & 34.3 & 14.1 & 18.2 & 9.1 & 5.1 & 1.0 \\
\hline
\end{tabular}

cambio en la mayor parte de los estudios, estas personas tienen alguna patología. Un alto porcentaje de artículos en $R L P F(87.1 \%)$ no tienen muestra, al igual que en las otras revistas analizadas, excepto IJCHP, que poseen un porcentaje significativo de artículos sin muestra/teóricos (véase Tabla 12).

Otros indicadores analizados fueron la temática general y la específica (véanse Tablas 13 y 14). La mayor parte de los estudios de IJCHP están relacionados con la patología (37.1\%), y en concreto, con la psicopatología (27.4\%). Lo mismo ocurre en Salud Mental con un $38.1 \%$ y un $31.1 \%$, respectivamente. En la $R L P F$, la mayor parte de las publicaciones también tienen relación con la patología (29.2\%), aunque de forma específica con el psicoanálisis (26.4\%). En la $R A C P$ predominan aquellos artículos relacionados con el asesoramiento y la orientación $(32.2 \%)$, sobre todo en aspectos que están relacionados con la psicoterapia (20\%). Igualmente, en Terapia Psicológica la mayor parte están relacionados con el asesoramiento y la orientación (24.2\%), aunque en este caso, además de centrarse en la psicoterapia $(15.2 \%)$, también tratan en la misma proporción la psicopatología (15.2\%).

\section{Discusión}

En este estudio se han analizado las cinco revistas más relevantes del ámbito de la Psicología Clínica y de la Salud, $\mathrm{y}$ han podido mostrarse las características principales de su producción científica, a través de indicadores considerados en la mayor parte de los estudios bibliométricos, por su gran importancia en la evolución de las revistas. Es importante resaltar que no se pretende demostrar si una revista es mejor que otra, simplemente se comentan aspectos que ayudan a tener una visión global de las mismas, y así conocer, por ejemplo, sobre qué temáticas se ha investigado más. 
Tabla 10. Distribución por países en función de la filiación institucional a la que pertenecen los autores.

\begin{tabular}{|c|c|c|c|c|c|}
\hline & IJCHP & RACP & RLPF & Salud Mental & Terapia Psicológica \\
\hline Total Países & 21 & 12 & 10 & 9 & 8 \\
\hline Alemania & $\mathrm{x}$ & $\mathrm{x}$ & & & \\
\hline Argentina & $\mathrm{x}$ & $\mathrm{x}$ & $\mathrm{x}$ & & \\
\hline Australia & $\mathrm{x}$ & $\mathrm{x}$ & & & $\mathrm{x}$ \\
\hline Bélgica & $\mathrm{x}$ & & & & \\
\hline Brasil & $\mathrm{x}$ & $\mathrm{x}$ & $\mathrm{x}$ & $\mathrm{x}$ & $\mathrm{x}$ \\
\hline Canadá & $\mathrm{x}$ & & & & \\
\hline Colombia & & $\mathrm{x}$ & & $\mathrm{x}$ & $\mathrm{x}$ \\
\hline Chile & $\mathrm{x}$ & $\mathrm{x}$ & & $\mathrm{x}$ & $\mathrm{x}$ \\
\hline China & $\mathrm{x}$ & & & & \\
\hline Corea & $\mathrm{x}$ & & & & \\
\hline \multicolumn{6}{|l|}{ El Salvador } \\
\hline EE.UU & $\mathrm{x}$ & & & $\mathrm{x}$ & $\mathrm{x}$ \\
\hline España & $\mathrm{x}$ & $\mathrm{x}$ & $\mathrm{x}$ & $\mathrm{x}$ & $\mathrm{x}$ \\
\hline Francia & & $\mathrm{x}$ & $\mathrm{x}$ & & \\
\hline Grecia & & $\mathrm{x}$ & & & \\
\hline Holanda & $\mathrm{x}$ & & & $\mathrm{x}$ & \\
\hline Irán & $\mathrm{x}$ & & & & \\
\hline Irlanda & $\mathrm{x}$ & & & & \\
\hline Italia & $\mathrm{x}$ & & $\mathrm{x}$ & & \\
\hline Lituania & & $\mathrm{x}$ & & & \\
\hline México & $\mathrm{x}$ & & $\mathrm{x}$ & $\mathrm{x}$ & $\mathrm{x}$ \\
\hline Malasia & & $\mathrm{x}$ & & & \\
\hline Perú & $\mathrm{x}$ & & $\mathrm{x}$ & & \\
\hline Polonia & $\mathrm{x}$ & $\mathrm{x}$ & & & \\
\hline Portugal & $\mathrm{x}$ & & $\mathrm{x}$ & & \\
\hline Reino Unido & $\mathrm{x}$ & & $\mathrm{x}$ & & \\
\hline Suiza & $\mathrm{x}$ & & & & \\
\hline Uruguay & & & $\mathrm{x}$ & & \\
\hline Venezuela & & & & $\mathrm{x}$ & \\
\hline
\end{tabular}

Nota. Los países están ordenados alfabéticamente. En cada revista se marca con una x el país de la filiación institucional de los autores que han publicado en ella.

Tabla 11. Porcentaje de artículos publicados en las revistas analizadas, en función de la metodología utilizada (2007-2011).

\begin{tabular}{|c|c|c|c|c|c|c|c|c|c|c|c|c|c|c|c|}
\hline & \multicolumn{3}{|c|}{ IJCHP } & \multicolumn{3}{|c|}{ RACP } & \multicolumn{3}{|c|}{ RLPF } & \multicolumn{3}{|c|}{ Salud Mental } & \multicolumn{3}{|c|}{ Terapia Psicológica } \\
\hline & 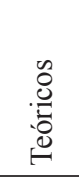 & 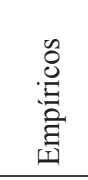 & 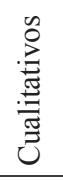 & $\begin{array}{l}0 \\
0 \\
0 \\
0 \\
0 \\
0 \\
0\end{array}$ & 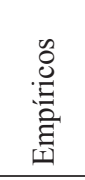 & 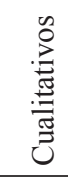 & $\begin{array}{l}n \\
0 \\
0 \\
0 \\
0 \\
0 \\
0 \\
0\end{array}$ & 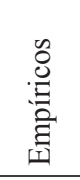 & 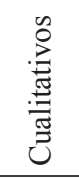 & $\begin{array}{l}0 \\
0 \\
0 \\
0 \\
0 \\
0 \\
0\end{array}$ & 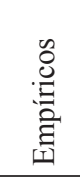 & 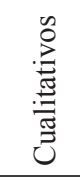 & 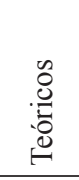 & 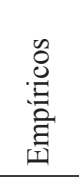 & 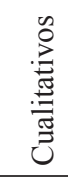 \\
\hline 2007 & 20.1 & 79.9 & 0 & 66.6 & 29.2 & 4.2 & 96.6 & 3.4 & 0 & 35.9 & 35.9 & 28.2 & 31.6 & 57.8 & 10.6 \\
\hline 2008 & 13.3 & 84.5 & 2.2 & 55.5 & 38.9 & 5.6 & 97.7 & 2.3 & 0 & 40.4 & 44.7 & 14.9 & 10.0 & 85.0 & 5.0 \\
\hline 2009 & 10.0 & 90.0 & 0 & 59.1 & 40.9 & 0 & 95.0 & 2.5 & 2.5 & 7.7 & 75.0 & 17.3 & 45.0 & 45.0 & 10.0 \\
\hline 2010 & 10.7 & 82.1 & 7.2 & 50.0 & 45.9 & 4.1 & 68.8 & 18.7 & 12.5 & 28.3 & 52.8 & 18.9 & 35.0 & 60.0 & 5.0 \\
\hline 2011 & 18.5 & 81.5 & 0 & 29.6 & 70.4 & 0 & 79.4 & 17.2 & 3.4 & 34.6 & 63.5 & 1.9 & 15.0 & 70.0 & 15.0 \\
\hline Total & 14.9 & 83.4 & 1.7 & 51.3 & 46.1 & 2.6 & 87.7 & 7.9 & 4.4 & 29.2 & 54.5 & 16.3 & 27.3 & 63.6 & 9.1 \\
\hline
\end{tabular}

Nota. IJCHP = International Journal of Clinical and Health Psychology; RACP = Revista Argentina de Clínica Psicológica; RLPF $=$ Revista Latinoamericana de Psicopatología Fundamental. 
En la actualidad, IJCHP tiene una evolución muy positiva en cuanto al incremento de su FI. Por otra parte, el progreso de la $R A C P$ y Salud Mental es estable. En cambio, en la $R L P F$ se ha producido un descenso a partir del año 2010.
Terapia Psicológica presenta un avance considerable, puesto que recientemente ha sido incorporada al JCR.

El número de citas totales que han obtenido estas revistas se corresponde con el FI, así como el idioma de publicación.

Tabla 12. Porcentaje de artículos publicados en las revistas analizadas, en función del tipo de muestra.

\begin{tabular}{lccccc}
\hline & IJCHP & RACP & RLPF & Salud Mental & $\begin{array}{c}\text { Terapia } \\
\text { Psicológica }\end{array}$ \\
\hline Tipo de muestra & & & $\%$ & & \\
\hline Revistas & 8.0 & 7.8 & 1.7 & 4.7 & 1.0 \\
Sin muestra/teórico & 1.7 & 42.6 & 87.1 & 28.4 & 28.3 \\
Familia/padres & 5.1 & 0 & 0 & 4.7 & 6.1 \\
Profesionales & 0.6 & 0 & 0 & 1.9 & 6.1 \\
Niños & 4.6 & 2.6 & 1.7 & 5.5 & 4.0 \\
Sin patología & 2.9 & 2.6 & 1.7 & 0.8 & 3.0 \\
Con patología & 1.7 & 0 & 0 & 4.7 & 1.0 \\
Adolescentes & 17.7 & 1.7 & 0.6 & 15.5 & 12.2 \\
Sin patología & 14.3 & 1.7 & 0.6 & 9.3 & 5.1 \\
Con patología & 3.4 & 0 & 0 & 6.2 & 7.1 \\
Adultos & 56.5 & 44.4 & 0 & 29.5 & 35.4 \\
Sin patología & 27.4 & 20.9 & 2.2 & 6.2 & 20.2 \\
Con patología & 29.1 & 23.5 & 5.6 & 23.3 & 15.2 \\
Ancianos & 4.0 & 0.9 & 0 & 1.2 & 2.0 \\
Otro & 1.7 & 0 & 1.1 & 8.6 & 5.1 \\
\hline
\end{tabular}

Nota. El tipo de muestra de los artículos analizados que no correspondía con ninguna de las categorías establecidas se clasificó como Otro. También se incluyeron en esta categoría aquellos artículos cuya muestra engloba más de una categoría.

IJCHP = International Journal of Clinical and Health Psychology; RACP = Revista Argentina de Clínica Psicológica; RLPF = Revista Latinoamericana de Psicopatología Fundamental.

Tabla 13. Porcentaje de artículos publicados en las revistas analizadas, en función de la temática general.

\begin{tabular}{|c|c|c|c|c|c|}
\hline & IJCHP & RACP & RLPF & Salud Mental & Terapia Psicológica \\
\hline Patología & 37.1 & 28.7 & 29.2 & 38.1 & 18.2 \\
\hline Psicología niño/adolescente & 8.6 & 2.6 & 3.4 & 2.3 & 1.0 \\
\hline Asesoramiento/orientación & 5.7 & 32.2 & 27.5 & 4.3 & 24.2 \\
\hline Psicopedagogía & 0 & 0 & 1.7 & 0 & 1.0 \\
\hline Evaluación y diagnóstico & 21.1 & 9.6 & 0 & 8.9 & 19.2 \\
\hline Psicología experimental & 7.4 & 4.3 & 5.6 & 13.6 & 3.0 \\
\hline Psicología general & 2.3 & 2.6 & 6.7 & 0.4 & 1.0 \\
\hline Psicología de la vejez & 1.1 & 0.9 & 1.1 & 1.2 & 0 \\
\hline Psicología industrial & 3.4 & 3.5 & 1.7 & 2.3 & 3.0 \\
\hline Parapsicología & 0 & 0.9 & 1.1 & 0 & 0 \\
\hline Personalidad & 2.3 & 8.7 & 5.6 & 0.8 & 4.0 \\
\hline Estudio psicológico social & 1.1 & 0.9 & 0 & 1.9 & 3.0 \\
\hline Psicofarmacología & 6.3 & 1.7 & 2.8 & 17.1 & 2.0 \\
\hline Psicología social & 1.1 & 3.5 & 6.2 & 0.8 & 7.1 \\
\hline Otras especialidades & 2.3 & 0 & 7.3 & 8.2 & 13.1 \\
\hline
\end{tabular}

Nota. IJCHP = International Journal of Clinical and Health Psychology; RACP = Revista Argentina de Clínica Psicológica; RLPF $=$ Revista Latinoamericana de Psicopatología Fundamental. 
Tabla 14. Porcentaje de artículos publicados en las revistas analizadas en función de la temática específica.

\begin{tabular}{|c|c|c|c|c|c|}
\hline & IJCHP & RACP & RLPF & Salud Mental & Terapia Psicológica \\
\hline Desórdenes del comportamiento & 8.0 & 15.7 & 11.2 & 6.2 & 1.0 \\
\hline Comportamiento desviado & 0.6 & 0.9 & 0 & 0 & 0 \\
\hline Discapacidad intelectual & 0.6 & 0 & 0 & 0.4 & 1.0 \\
\hline Psicopatología & 27.4 & 14.8 & 18.5 & 31.1 & 15.2 \\
\hline Psicología evolutiva & 3.4 & 1.7 & 1.7 & 1.2 & 1.0 \\
\hline Problemas de aprendizaje & 3.4 & 0 & 0.6 & 0 & 0 \\
\hline Psicología escolar & 4.6 & 0.9 & 0.6 & 0 & 0 \\
\hline Patología del lenguaje & 0 & 0 & 0.6 & 0.8 & 0 \\
\hline Terapia del comportamiento & 3.4 & 7.8 & 0 & 0.8 & 3.0 \\
\hline Psicología de la orientación & 0.6 & 0.6 & 0 & 0.4 & 0 \\
\hline Asesoramiento y orientación ed. & 0.6 & 1.7 & 0 & 0.8 & 0 \\
\hline Terapia de grupo & 0 & 0.9 & 0 & 0 & 6.1 \\
\hline Psicoanálisis & 0 & 0.9 & 26.4 & 0.4 & 0 \\
\hline Psicoterapia & 2.9 & 20.0 & 2.2 & 1.6 & 15.2 \\
\hline Procesos cognitivos & 0 & 0 & 0 & 0 & 1.0 \\
\hline Métodos educativos & 0 & 0 & 1.1 & 0 & 0 \\
\hline Psicología diferencial & 2.3 & 0.9 & 0 & 0 & 0 \\
\hline Teoría de la medición & 0.6 & 0 & 0 & 0.4 & 0 \\
\hline Estadística & 5.1 & 4.3 & 0 & 0 & 1.0 \\
\hline Psicometría & 3.4 & 1.7 & 0 & 1.9 & 8.1 \\
\hline Análisis de la escala & 1.7 & 0.9 & 0 & 0 & 0 \\
\hline Elaboración de tests & 0 & 0 & 0 & 0.8 & 0 \\
\hline Validez de tests & 6.9 & 1.7 & 0 & 5.8 & 10.1 \\
\hline Actividad cerebral & 1.1 & 0 & 0.6 & 8.2 & 0 \\
\hline Psicología comparada & 0 & 0 & 0 & 1.2 & 0 \\
\hline Emoción & 0 & 0 & 0 & 0 & 2.0 \\
\hline Análisis experimental conducta & 0 & 0 & 0 & 0 & 1.0 \\
\hline Niveles de actividad & 0.6 & 0 & 0 & 0 & 0 \\
\hline Procesos de la memoria & 0 & 0 & 1.1 & 1.2 & 0 \\
\hline Procesos mentales & 0 & 0 & 1.1 & 0 & 0 \\
\hline Psicología fisiológica & 0 & 0 & 0 & 3.1 & 0 \\
\hline Reacción. reflejos & 6.3 & 4.3 & 1.1 & 0 & 0 \\
\hline Procesos sensoriales & 0 & 0 & 0.6 & 0 & 0 \\
\hline Muerte & 0.6 & 0.9 & 0.6 & 0 & 0 \\
\hline Madurez & 0 & 0 & 0 & 0.4 & 0 \\
\hline Senectud & 0 & 0.9 & 1.1 & 0.8 & 0 \\
\hline Prevención de accidentes & 1.1 & 0 & 0 & 0.8 & 0 \\
\hline Motivación y actitudes & 1.7 & 0 & 1.1 & 1.2 & 2.0 \\
\hline Planificación puestos de trabajo & 0.6 & 0.9 & 0 & 0 & 0 \\
\hline Comportamiento organización & 0.6 & 0 & 0.6 & 0 & 0 \\
\hline Selección de personal & 0.6 & 1.7 & 0 & 0 & 0 \\
\hline Evaluación del rendimiento & 0 & 0 & 0 & 0.4 & 1.0 \\
\hline Percepción extrasensorial & 0 & 0 & 1.1 & 0 & 0 \\
\hline Hipnosis & 0 & 0.9 & 0 & 0 & 0 \\
\hline
\end{tabular}




\begin{tabular}{|c|c|c|c|c|c|}
\hline & IJCHP & RACP & RLPF & Salud Mental & Terapia Psicológica \\
\hline Creatividad & 0 & 0 & 0.6 & 0 & 0 \\
\hline Cultura y personalidad & 0 & 0 & 0 & 0.4 & 1.0 \\
\hline Desarrollo de la personalidad & 0 & 0 & 2.8 & 0.4 & 2.0 \\
\hline Medida de la personalidad & 1.1 & 5.2 & 1.1 & 0 & 0 \\
\hline Estructura de la personalidad & 0.6 & 0 & 1.1 & 0 & 1.0 \\
\hline Teoría de la personalidad & 0.6 & 3.5 & 0 & 0 & 0 \\
\hline Discriminación & 0 & 0 & 0 & 1.2 & 3.0 \\
\hline Grupos minoritarios & 1.1 & 0.9 & 0 & 0.8 & 0 \\
\hline Alcoholismo & 0 & 0 & 0 & 5.8 & 0 \\
\hline Reacciones del comportamiento & 0 & 0 & 0 & 0.4 & 0 \\
\hline Abuso de drogas & 4.6 & 0 & 1.1 & 8.6 & 2.0 \\
\hline Efecto de las drogas & 0.6 & 0 & 1.1 & 0.4 & 0 \\
\hline Tratamiento de la drogadicción & 0 & 0.9 & 0 & 1.9 & 0 \\
\hline Actitudes & 0 & 0 & 0 & 0.4 & 2.0 \\
\hline Comportamiento colectivo & 1.1 & 0.9 & 1.7 & 0 & 0 \\
\hline Psicología comunitaria & 0 & 0.9 & 2.2 & 0 & 4.0 \\
\hline Resolución de conflictos & 0 & 0 & 0 & 0 & 1.0 \\
\hline Psicología forense & 0 & 0 & 0 & 0.4 & 0 \\
\hline Marketing & 0 & 0 & 0.6 & 0 & 0 \\
\hline Comportamiento político & 0 & 0 & 1.1 & 0 & 0 \\
\hline Opinión pública & 0 & 0 & 0.6 & 0 & 0 \\
\hline Otras & 0 & 0 & 7.9 & 9.3 & 14.1 \\
\hline
\end{tabular}

Nota. IJCHP = International Journal of Clinical and Health Psychology; RACP = Revista Argentina de Clínica Psicológica; RLPF $=$ Revista Latinoamericana de Psicopatología Fundamental.

Según Buela-Casal y Zych (2012b), las publicaciones en inglés reciben más citas que las que están escritas en español, aunque no sean de mayor calidad. De ahí que la revista analizada con mayor FI y con mayor número de citas recibidas sea IJCHP, en la que más de la mitad de sus artículos están publicados en lengua inglesa (e.g., Escartin, Rodríguez-Carballeira, Gómez-Benito y Zapf, 2010; Griffin, Guerin, Sharry y Drumm, 2010). La RACP en los últimos cinco años ha publicado la totalidad de sus artículos en español, no tiene un elevado número de citas, aunque sí cuenta con el mayor índice de inmediatez entre ellas. A pesar de que Salud Mental tiene un número de citas considerable, su FI es menor que el de Terapia Psicológica, aun teniendo la primera más citas. Aunque este índice puede depender, entre otros, de la categoría en la que está insertada la revista en el JCR, también puede influir el número de años de inclusión en la WoS, debido al aumento de la visibilidad de las publicaciones. Por otro lado, la probabilidad de recibir citas puede aumentar cuando el número de artículos publicados por número es mayor. Este es el caso de Salud Mental, cuyo número de citas totales puede estar relacionado con sus casi tres décadas en la WoS y por el número de artículos publicados, que supera el de todas las revistas analizadas.

Según Camps (2008), los hábitos de publicación y la productividad son distintos entre las áreas científicas y también entre las disciplinas que pertenecen a un área. En este caso, Salud Mental pertenece al grupo de la revistas de Psiquiatría, categoría en la que el FI es más elevado que el de Psicología Clínica, donde están incluidas el resto de revistas. Tal y como afirman Velasco, Eiros, Pinilla y San Román (2012), debe considerarse el área temática en la que están incluidas las revistas a la hora de evaluarlas.

Por otra parte, el bajo índice de impacto y número de citas de la $R L P F$ podría confirmarse con la publicación de la mayor parte de sus artículos en portugués, ya que es un idioma menos difundido que el inglés o el español. En este caso, una mínima parte de los artículos están publicados en estos idiomas, por lo que se restringe el acceso a los lectores del resto de la comunidad iberoamericana y del mundo. Esto también repercute en el índice de inmediatez, ya que si los investigadores no pueden leerlos dada la dificultad que puede suponer el idioma, tampoco pueden citarlos. 
En cuanto al índice de autoría, cabe destacar que la mayor parte de las aportaciones son de un autor en la $R L P F(e . g$., França, 2009; Oliveira, 2011) y en la $R A C P$ (e.g., Keegan, 2007; Ravazzola, 2010). En cambio, predominan los artículos de autoría compartida en Terapia Psicológica (e.g., Pina, Sánchez-Sosa, Fierros, Ybarra y Cazares, 2011; Zúñiga, Vinet y León, 2011), IJCHP (e.g., Pascual, Etxebarria, Cruz y Echeburúa, 2011; Watson, Clark y Stasik, 2011) y Salud Mental (e.g., Salazar, Martínez y Barrientos, 2009; Saldívar y Romero, 2009). Según Quevedo-Blasco y LópezLópez (2010), se podría afirmar que cuando el número de autores firmantes es menor, como en la $R L P F$, la $R A C P$ y Terapia Psicológica, puede ser debido a que realmente todos ellos han trabajado en el artículo, o también puede ser debido a otra causa y podría darse la situación de que exista poca colaboración entre los miembros en los grupos de investigación.

Respecto a la filiación institucional de los autores, se demuestra que existen contribuciones que pertenecen al mismo país de las revistas analizadas (e.g., Díaz-Anzaldúa, Díaz-Martínez y Díaz-Martínez, 2011; Micin y Bagladi, 2011; Teixeira, 2011) y aportaciones de autores extranjeros (e.g., Alves y Pereira, 2011; García-García, Rosa-Alcázar y Olivares-Olivares, 2011; Vinaccia y Quiceno, 2011), aspecto que es muy positivo para las revistas en el incremento de su nivel de internacionalidad (Buela-Casal y Zych, 2012a, 2012b). Según Quevedo-Blasco y López-López (2010), las contribuciones internacionales indican que existe colaboración entre autores e instituciones de distintos países.

Otro indicador analizado fue la metodología de los artículos. Un dato revelador es que las revistas que tienen un mayor número de citas son aquellas en las que predominan los artículos empíricos. Sin embargo, en el estudio de BuelaCasal et al. (2009) se comparan los tipos de artículos más citados en revistas españolas de psicología y se sostiene que los estudios teóricos reciben más citas que los empíricos. Esto puede deberse a que las revistas analizadas en dicho estudio tratan temas generales con un enfoque multidisciplinar. Un pequeño porcentaje de las publicaciones sigue una metodología cualitativa en todas las revistas analizadas. A pesar de esto, se puede comprobar que las revistas latinoamericanas poseen un porcentaje mayor de artículos con esta metodología que la revista española. Esto se puede explicar por la consolidada tradición en investigación sobre Psicología Social/Comunitaria y en Psicoanálisis existente en Latinoamérica. Aun así, el porcentaje de artículos cualitativos es bajo, quizás porque, según Castillo y Vásquez (2003), las normas para evaluar la calidad de este tipo de artículos son menos conocidas.

La mayor parte de los estudios de todas las revistas, excepto la $R L P F$ en la que predominan los estudios sin muestra (e.g., Alves y Andersen, 2011; Rezende, 2011), tienen como participantes en las investigaciones a personas adultas. Así, en IJCHP destacan trabajos como el de
Rojas-Vilches, Negy y Reig-Ferrer (2011) y Watson et al. (2011); el de Kirszman y Salgueiro (2010) y Tartaglini, Ofman y Stefani (2010) en la $R A C P$; el de Fresán, Minaya, Cortés-López y Ugalde (2011) y Vázquez, González-Macías, Berlanga y Aedo (2011) en Salud Mental; y el de Pimentel y Cova (2011) y Rivera, Cruz y Muñoz (2011) en Terapia Psicológica. Estas revistas no están especializadas en grupos de población concretos, por tanto, los estudios con adultos puede que sean los que provocan mayor interés. Además, las temáticas más representativas que se publican en ellas están relacionadas con alguna patología o psicopatología y la psicoterapia, puesto que pertenecen al ámbito de la psicología clínica y de la salud. Así, se pueden destacar estudios como el de Beutel, Bleichner, Von Heymann, Tritt y Hardt (2011) en IJCHP, el de Gercovich et al. (2011) en la RACP, el de Moreira (2011) en la RLPF, el de Alonso-Fernández (2011) en Salud Mental o el de Lecannelier, Ascanio, Flores y Hoffmann (2011) en Terapia Psicológica.

La finalidad de las publicaciones periódicas es aportar conocimiento científico de la disciplina y difundirlo. Entre otras, la prueba de que este conocimiento ha sido difundido es la obtención de citas, por tanto, es interesante comprobar qué temáticas son las más citadas. Por ejemplo, en IJCHP los temas más citados en la WoS son psicología general, evaluación y diagnóstico en psicología y patología. La mayor parte de los artículos publicados pertenecen a las dos últimas temáticas, sin embargo, solo el $2.3 \%$ de sus artículos forman parte del ámbito de la psicología general. En el caso de la $R A C P$, el $32.2 \%$ de sus artículos tratan el asesoramiento y la orientación, pero las temáticas más citadas en la WoS son patología, psicología social y parapsicología.

Por su parte, la $R L P F$ tiene como temáticas más citadas, psicología general, psicología social y patología, coincidiendo solo esta última con un mayor número de artículos publicados. Salud Mental publica la mayor parte de sus artículos sobre patología, en cambio, esta no es la temática más citada que corresponde a psicofarmacología, y evaluación y diagnóstico en psicología. Por último, las temáticas más tratadas en Terapia Psicológica son el asesoramiento y la orientación, la evaluación y el diagnóstico en psicología, y la psicología social. Éstas coinciden con las de aquellos artículos que tienen un mayor número de citas en la WoS, a excepción de psicología social que es una temática citada y solo el $7.1 \%$ de las publicaciones se encuadran en ella.

Una vez conocidas las características de estas revistas se puede afirmar que su evolución es positiva. Además, sería interesante considerar aspectos como la publicación de artículos relacionados con las temáticas más citadas o incluir una versión en lengua inglesa de los artículos para lograr una mayor difusión fuera de los países de habla hispana, ya que según Zych y Buela-Casal (2007), la ciencia está dominada por el mundo anglosajón. También sería necesario conseguir un incremento de autores pertenecientes a instituciones extranjeras y de distintas nacionalidades, con 
el fin de obtener un enriquecimiento del conocimiento y una mayor colaboración entre países.

\section{Referencias}

Agudelo, D., Buela-Casal, G., y Bretón- López, J. (2003). Análisis bibliométrico de las revistas de Psicología Clínica editadas en castellano. Psicothema, 15, 507-516.

Agudelo, D., Buela-Casal, G., y Bretón-López, J. (2004). Análisis bibliométrico de las revistas relacionadas con la Psicología de la Salud editadas en castellano. Salud Mental, 27, 70-85.

Alonso-Fernández, F. (2011). La alexitimia y su trascendencia clínica y social. Salud Mental, 34, 481-490.

Alves, N., y Andersen, C. (2011). Psicanálise e antropologia: diálogos possíveis. Revista Latinoamericana de Psicopatología Fundamental, 14, 57-69.

Alves, T.G., y Pereira, M.G. (2011). Physiological variables and burden in adult children of cancer patients. Revista Argentina de Clínica Psicológica, 20, 255-263.

Alvis-Guzmán, N., y De La Hoz-Restrepo, F. (2002). Producción Científica en Ciencias de la Salud en Colombia, 1993-2003. Revista de Salud Pública, 8, 27-37.

Beutel, M.E., Bleichner, F., Von Heymann, F., Tritt, K., y Hardt, J. (2011). Inpatient psychosomatic treatment of anxiety disorders: Comorbidities, predictors, and outcomes. International Journal of Clinical and Health Psychology, 11, 443-457.

Buela-Casal, G. (2003). Evaluación de la calidad de los artículos y de las revistas científicas: propuesta del factor impacto ponderado y de un índice de calidad. Psicothema, 15, 23-35.

Buela-Casal, G. (2010). Índices de impacto de las revistas científicas e indicadores para medir el rendimiento de los investigadores. Revista de Psicodidáctica, 15, 3-19.

Buela-Casal, G., Bermúdez, M.P., Sierra, J. C., Quevedo-Blasco, R., y Castro, A. (2010). Ranking de 2009 en investigación de las universidades públicas españolas. Psicothema, 22, 171-179.

Buela-Casal, G., Bermúdez, M.P., Sierra, J.C., Quevedo-Blasco, R., Castro, A., y Guillén-Riquelme, A. (2011). Ranking de 2010 en investigación de las universidades públicas españolas. Psicothema, 23, 527-536.

Buela-Casal, G., Bermúdez, M.P., Sierra, J. C., Quevedo-Blasco, R., Castro, A., y Guillén-Riquelme, A. (2012). Ranking de 2011 en producción y productividad en investigación de las universidades públicas españolas. Psicothema, 24, 505-515.

Buela-Casal, G., Bermúdez, M.P., Sierra, J. C., Quevedo-Blasco, R., Guillén-Riquelme, A., y Castro, A. (2010). Relación de la productividad científica y eficiencia en investigación con la financiación de las comunidades autónomas españolas. Psicothema, 22, 924-931.

Buela-Casal, G., Bermúdez, M.P., Sierra, J. C., Quevedo-Blasco, R., Guillén-Riquelme, A., y Castro, A. (2012). Productividad y eficiencia en investigación de 2010: relación con la financiación de las comunidades autónomas españolas. Revista Electrónica de Metodología Aplicada (REMA), 17, 35-50.

Buela-Casal, G., Olivas-Ávila, J., Musi-Lechuga, B., y Zych, I. (2011). The $h$ index of the presidents of the American Psychological Association (APA) through journal articles included in the Web of Science. International Journal of Clinical and Health Psychology, 11, 95-107.

Buela-Casal, G., Perakakis, P., Taylor, M., y Checa, P. (2006). Measuring Internationality: Reflections and perspectives on academic journals. Scientometrics, 67, 45-65. doi:10.1007/s11192-006-0050-Z

Buela-Casal, G., y Zych, I. (2012a). How to measure the internationality of scientific publications. Psicothema, 24, 435-441.

Buela-Casal, G., y Zych, I. (2012b). What do the scientists think about the impact factor? Scientometrics, 92, 281-292. doi: 10.1007/s11192012-0676-y

Buela-Casal, G., Zych, I., Medina, A., Viedma del Jesús, M.I., Lozano, S., y Torres, G. (2009). Analysis of the influence of the two types of the journal articles; theoretical and empirical on the impact factor of a journal. Scientometrics, 80, 265-282. doi: 10.1007/s11192-008-1715-6

Camps, D. (2008). Limitaciones de los indicadores bibliométricos en la evaluación de la actividad científica biomédica. Colombia Médica. 39, 74-79.

Castillo, E., y Vásquez, M.L. (2003). El rigor metodológico en la investigación cualitativa. Colombia Médica, 34, 164-167.

Cortada de Kohan, N. (2002). Importancia de la investigación psicométrica. Revista Latinoamericana de Psicología, 34, 229-240.

Díaz-Anzaldúa, A., Díaz-Martínez, A., y Díaz-Martínez, L.R. (2011). The complex interplay of genetics, epigenetics, and environment in the predisposition to alcohol dependence. Salud Mental, 34, 157-166.

Escartin, J., Rodríguez-Carballeira, A., Gómez-Benito, J., y Zapf, D. (2010). Development and validation of the workplace bullying scale EAPA-T. International Journal of Clinical and Health Psychology, 10, 519-539.

França, O. (2009). Por uma nova concepção de universal. Revista Latinoamericana de Psicopatología Fundamental, 12, 650-661.

Fresán, A., Minaya, O., Cortés-López, J.L., y Ugalde, O. (2011). Características demográficas y clínicas asociadas a la dependencia a benzodiacepinas en pacientes psiquiátricos. Salud Mental, 34, 103-109.

Gallegos, M. (2010). La Revista Latinoamericana de Psicología en sus 40 años de historia: 1969-2009. Universitas Psychologica, 9, 911-924.

García-García, E.S., Rosa-Alcázar, A. I., y Olivares-Olivares, P.J. (2011). Terapia de exposición mediante realidad virtual e internet en el trastorno de ansiedad/fobia social: una revisión cualitativa. Terapia Psicológica, 29, 233-243.

García-Sílberman, S., Arana, D., Martínez, R., Infante, R., y Jiménez, A. (2004). La investigación sobre aspectos epidemiológicos y psicosociales de la salud mental: un análisis bibliométrico. Salud Mental, 27, 8-22.

Garfield, E. (2003). The meaning of the Impact Factor. International Journal of Clinical and Health Psychology, 3, 363-369.

Gercovich, D., Torrente, F., López, P., Margiolakis, P., Bortolato, D., Hirsch, H., y Gil Deza, E. (2011). Psicoterapia focalizada en pacientes oncológicos. Resultados del tratamiento en un contexto naturalístico. Revista Argentina de Clínica Psicológica, 3, 239-246.

Gómez-García, A., Ramiro, M.T., Ariza, T., y Granados, M.R. (2012). Estudio bibliométrico de Educación XXI. Educación XXI, 15, 17-41.

Granados, M.R., Ariza, T., Gómez-García, A., y Ramiro, M.T. (2011). Estudio bibliométrico de Aula Abierta. Aula Abierta, 39, 97-111.

Griffin, C., Guerin, S., Sharry, J., y Drumm, M. (2010). A multicentre controlled study of an early intervention parenting programme for young children with behavioural and developmental difficulties. International Journal of Clinical and Health Psychology, 10, 279-294.

Hartley, J. (2012). New ways of making academic articles easier to read. International Journal of Clinical and Health Psychology, 12, 143-160.

Keegan, E. (2007). La ansiedad generalizada como fenómeno: criterios diagnósticos y concepción psicopatológica. Revista Argentina de Clínica Psicológica, 16, 49-56.

Kirszman, D., y Salgueiro, M.C. (2010). Parejas y familias en la práctica clínica. Algunos lineamientos para la formación y el entrenamiento. Revista Argentina de Clínica Psicológica, 19, 41-43.

Lecannelier, F., Ascanio, L., Flores, F., y Hoffmann, M. (2011). Apego y Psicopatología: Una Revisión Actualizada Sobre los Modelos Etiológicos Parentales del Apego Desorganizado. Terapia Psicológica, $29,107-116$.

Micin, S., y Bagladi, V. (2011). Salud Mental en Estudiantes Universitarios: Incidencia de Psicopatología y Antecedentes de Conducta Suicida en Población que Acude a un Servicio de Salud Estudiantil. Terapia Psicológica, 29, 53-64.

Moreira, V. (2011). O inconsciente no pensamento de Merleau-Ponty: contribuição para a psicoterapia. Revista Latinoamericana de Psicopatología Fundamental, 14, 110-112.

Navarrete-Cortés, J., Fernández-López, J. A., López-Baena, A., QuevedoBlasco, R., y Buela-Casal, G. (2010). Global psychology: a bibliometric analysis of Web of Science publications. Universitas Psychologica, 9, 553-567. 
Navarrete-Cortés, J., Quevedo-Blasco, R., Chaichio-Moreno, J.A., Ríos, C., y Buela-Casal, G. (2009). Análisis cuantitativo por países de la productividad en psicología de las revistas en la Web of Science. Revista Mexicana de Psicología, 26, 131-143.

Olivas-Ávila, J.A., y Musi-Lechuga, B. (2012). Aprendiendo a buscar en la Web of Science: réplica al estudio de Aluja, Becoña, Botella, Colom, Echeburúa, Forns, Pérez y Vila (2011). Psicothema, 24, 594-602.

Oliveira, C. (2011). Eugenizar a alma: a constituição da euphrenia no projeto de higiene mental voltado à infância da Liga Brasileira de Hygiene Mental. Revista Latinoamericana de Psicopatología Fundamental, 14, 627-641.

Ortiz, J., y Vera-Villarroel, P. (2003). Investigaciones en psicología clínica basadas en la evidencia en Chile: un análisis bibliométrico de tres revistas de Psicología. Terapia Psicológica, 21, 61-66.

Pascual, A., Etxebarria, I., Cruz, M.S., y Echeburúa, E. (2011). Las variables emocionales como factores de riesgo de los trastornos de la conducta alimentaria. International Journal of Clinical and Health Psychology, 11, 229-247.

Pimentel, M., y Cova, F. (2011). Efectos de la Rumiación y la Preocupación en el Desarrollo de Sintomatología Depresiva y Ansiosa en Estudiantes Universitarios de la Ciudad de Concepción, Chile. Terapia Psicológica, 29, 43-52.

Pina, J.A., Sánchez-Sosa, J.J., Fierros, L. E., Ybarra, J.L., y Cazares, O. (2011). Variables psicológicas y adhesión en personas con VIH: evaluación en función del tiempo de infección. Terapia Psicológica, 29, 149-157.

Pritchard, A. (1969). Statistical bibliography or bibliometrics? Journal of Documentation, 25, 348-349.

Quevedo-Blasco, R., Ariza, T., y Raya, L. (2012). Análisis de la producción de la Psicología Jurídica en España (1989-2010). Aula Abierta, 40, 117-128.

Quevedo-Blasco, R., Díaz-Piedra, C., y Guglielmi, O. (2010). Análisis comparativo de las publicaciones sobre drogodependencias en las revistas de Psicología Clínica y Psiquiatría iberoamericanas indexadas en el Journal Citation Reports. Salud Mental, 33, 133-143.

Quevedo-Blasco, R., y López-López, W. (2010). Análisis bibliométrico de las revistas multidisciplinares de psicología recientemente incorporadas en la Web of Science (2008-2009). Psicologia: Reflexão e Crítica, 23, 384-408.

Quevedo-Blasco, R., y López-López, W. (2011). Situación de las revistas iberoamericanas de psicología en el Journal Citation Reports de 2010. Universitas Psychologica, 10, 937-947.

Ravazzola, M.C. (2010). ¿Importan las diversidades de género en las decisiones de los terapeutas? Revista Argentina de Clínica Psicológica, 19, 83-89.

Rezende, M. (2011). Das neuroses atuais às neuroses traumáticas: continuidade e ruptura. Revista Latinoamericana de Psicopatología Fundamental, 14, 70-82.

Rivera, D., Cruz, C., y Muñoz, C. (2011). Satisfacción en las Relaciones de Pareja en la Adultez Emergente: El Rol del Apego, la Intimidad y la Depresión. Terapia Psicológica, 29, 77-83.

Rojas-Vilches, A. P., Negy, C., y Reig-Ferrer, A. (2011). Attitudes toward seeking therapy among Puerto Rican and Cuban American young adults and their parents. International Journal of Clinical and Health Psychology, 11, 313-341.
Salazar, M.L., Martínez, K.I., y Barrientos, V. (2009). Evaluación de un componente de inducción al tratamiento con adolescentes usuarios de alcohol del Distrito Federal. Salud Mental, 32, 469-477.

Saldívar, G., y Romero, M.P. (2009). Reconocimiento y uso de tácticas de coerción sexual en hombres y mujeres en el contexto de relaciones heterosexuales. Un estudio en estudiantes universitarios. Salud Mental, 32, 487-494.

Tartaglini, M.F., Ofman, S.D., y Stefani, D. (2010). Sentimiento de Sobrecarga y Afrontamiento en Cuidadores Familiares Principales de Pacientes con Demencia. Revista Argentina de Clínica Psicológica, 19, 221-226.

Teixeira, M. (2011). Os experimentos da Guatemala: infecção intencional de presos, soldados e insanos pela Laboratório de Pesquisa em Doneça Venérea dos EUA. Revista Latinoamericana de Psicopatología Fundamental, 14, 690-698.

UNESCO (1988). Proyecto de nomenclatura internacional normalizada relativa a la Ciencia y la Tecnología. Disponible desde http://unesdoc. unesco.org/images/0008/000829/082946sb.pdf

Vázquez, R.D., González-Macías, L., Berlanga, C., y Aedo, F.J. (2011). Effect of acupuncture treatment on depression: Correlation between psychological outcomes and salivary cortisol levels. Salud Mental, $34,21-26$

Velasco, B., Eiros, J. M., Pinilla, J.M., y San Román, J.A. (2012). La utilización de indicadores bibliométricos para evaluar la actividad investigadora. Aula Abierta, 40, 75-84.

Vera-Villarroel, P., y Mustaca, A. (2006). Investigaciones en Psicología Clínica basadas en la evidencia en Chile y Argentina. Revista Latinoamericana de Psicología, 38, 551-565.

Villalobos-Galvis, F., y Puertas-Campanario, R. (2007). Impacto e internacionalidad de tres revistas iberoamericanas en revistas de Psicología de España. Revista Latinoamericana de Psicología, 39, 593-608.

Villar, A. (2011). El "eigenfactor": un nuevo y potente instrumento bibliométrico para evaluar la investigación. Aula Abierta, 39, 85-96.

Vinaccia, S., y Quiceno, J.M. (2011). Calidad de Vida Relacionada con la Salud y Factores Psicológicos: Un Estudio desde la Enfermedad Pulmonar Obstructiva Crónica - EPOC. Terapia Psicológica, 29, 65-75.

Watson, D., Clark, L.A., y Stasik, S.M. (2011). Emotions and the emotional disorders: A quantitative hierarchical perspective. International Journal of Clinical and Health Psychology, 11, 429-442.

Zubeidat, I, Desvarieux, A.R., Salamanca, Y., y Sierra, J.C. (2004). Análisis bibliométrico de la revista Journal of Sex Research. Universitas Psychologica, 3, 47-54.

Zúñiga, D., Vinet, E.V., y León, E. (2011). Caracterización Psicométrica del Psychopathy Checklist: Youth Version (PCL: YV) en Adolescentes Chilenos. Terapia Psicológica, 29, 25-31.

Zych, I., y Buela-Casal, G. (2007). Índice de internacionalidad de las revistas iberoamericanas de Psicología incluidas en la Web of Science. Revista Mexicana de Psicología, 24, 15-22.

Zych, I., y Buela-Casal, G. (2010). Internacionalidad de las revistas de Psicología Multidisciplinar editadas en Iberoamérica e incluidas en la Web of Science. Universitas Psychologica, 9, 27-34.

Zych, I., y Quevedo-Blasco, R. (2011). A decade of the International Journal of Clinical and Health Psychology (2001-2010). International Journal of Clinical and Health Psychology, 11, 549-561. 